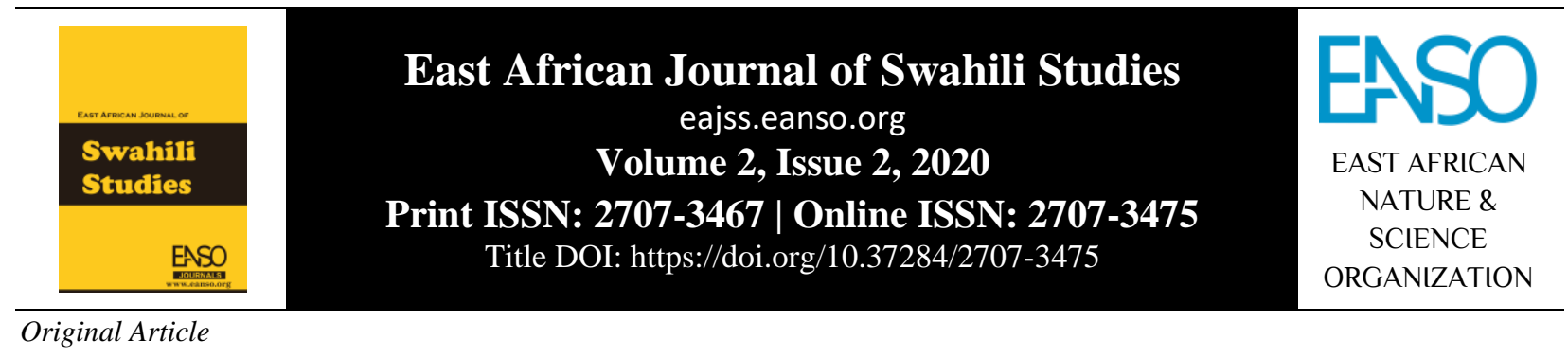

\title{
Sifa za Kimuundo Katika Diwani Huru ya Rangi ya Anga
}

\author{
Eunice Wavinya Nzioki ${ }^{1 *} \&$ Prof. Geoffrey Kitula King' ${ }^{1}{ }^{1}$ \\ ${ }^{1}$ Chuo Kikuu cha Kenyatta, S. L. P 43844 - 00100, Nairobi, Kenya. \\ *Barua pepe ya mawasiliano: mwinzieunice@gmail.com.
}

DOI ya Nakala: https://doi.org/10.37284/eajss.2.2.237

\section{Tarehe ya Uchapishaji: IKISIRI}

15 Novemba 2020 Makala hii inalenga kuchambua na kutathmini sifa za kimuundo katika diwani huru ya Rangi ya Anga. Sifa za kimuundo ni muhimu katika kuudhibiti usomaji

Istilahi Muhimu: wa mashairi teule na kumwelekeza msomaji katika kuuelewa ushairi.

Sifa za Kimuundo, Ijapokuwa tafiti nyingi zimefanywa kuhusu muundo katika mashairi, pana haja

Kimofolojia,

Kisintaksia,

Wizani, ya kuchambua sifa za kimuundo katika ushairi huru hasa diwani huru ya Rangi ya Anga. Utafiti huu ulitumia nadharia changamano: nadharia ya uhakiki ya umuundo na nadharia ya uhakiki wa kimtindo. Nadharia ya uhakiki ya Ushairi Huru. umuundo iliasisiwa na Ferdinand De Saussure karne ya ishirini. Mawazo yake yaliendelezwa na Culler (1975) aliyeangazia umuundo katika ushairi. Alisisitiza kwamba ushairi ni ishara zinazojisimamia bila kudhibitiwa na mtunzi na ufasiri wa maana unatokana na namna vipengee vya kimuundo huchagizana kimaana. Kutokana na nadharia hii kutokuwa na urejelezi wa nje, nadharia ya uhakiki wa kimtindo ilitumika kufidia udhaifu huu. Utafiti huu uliongozwa na nadharia ya uhakiki wa kimtindo iliyoasisiwa na Coombes (1953) na kuendelezwa na Leech (1969). Mihimili mitatu ilitumika kuuongoza utafiti. Mathalan, viwango vya kimofolojia na kisintaksia ni msingi mkuu wa lugha unaodhibiti matumizi ya lugha, matumizi ya maumbo ya picha huonyesha umilisi wa mtunzi katika kuelezea hisia zake na vipashio mbalimbali vya muundo huingiliana na kujengana. Utafiti huu ulitumia sampuli ya kimaksudi kuteua diwani huru ya Rangi ya Anga (2014) kutokana na matumizi ya miundo tengemano na wepesi wa mawazo ya mtunzi. Uhakiki wa sifa za kimuundo ulizingatia sampuli ya kimaksudi ya mashairi teule, tasnifu, majarida na tahakiki. Data ya pili ilijumuisha mahojiano na mtunzi Kithaka wa Mberia. Matokeo ya data yaliwasilishwa kwa njia ya maelezo. Yalionyesha kuwa sifa za kimuundo ni za kimsingi katika utunzi wa mashairi teule, ujenzi wa wizani na kudhibiti usomaji. Utafiti huu unapendekeza 
wasomaji wa ushairi huru wazingatie sifa za kimuundo ili kuufasiri ujumbe ipasavyo.

\section{APA CITATION}

Nzioki, E., \& King'ei, G. (2020). Sifa za Kimuundo Katika Diwani Huru ya Rangi ya Anga. East African Journal of Swahili Studies, 2(2), 153-167. https://doi.org/10.37284/eajss.2.2.227

\section{CHICAGO CITATION}

Nzioki, Eunice, and Geoffrey King'ei. 2020. "Sifa za Kimuundo Katika Diwani Huru ya Rangi ya Anga". East African Journal of Swahili Studies 2 (2), 153-167. https://doi.org/10.37284/eajss.2.2.237.

\section{HARVARD CITATION}

Nzioki, E. and King'ei, G. (2020) "Sifa za Kimuundo Katika Diwani Huru ya Rangi ya Anga”, East African Journal of Swahili Studies, 2(2), pp. 153-167. doi: 10.37284/eajss.2.2.237.

\section{IEEE CITATION}

E. Nzioki, and G. King'ei, "Sifa za Kimuundo Katika Diwani Huru ya Rangi ya Anga”, EAJSS, vol. 2, no. 2, pp. 153-167, Nov. 2020.

\section{MLA CITATION}

Nzioki, Eunice, and Geoffrey King'ei. 2020. "Sifa za Kimuundo Katika Diwani Huru ya Rangi ya Anga". East African Journal of Swahili Studies, Vol. 2, no. 2, Nov. 2020, pp. 153-167, doi:10.37284/eajss.2.2.237.

\section{UTANGULIZI}

Kithaka wa Mberia, katika diwani huru ya Rangi ya Anga ameibuka na miundo mipya na ya kipekee katika kuwasilisha kazi yake kwa wasomaji. Ijapokuwa tafiti nyingi zimefanywa kuhusu muundo katika ushairi, nyingi zimeangazia muundo katika ushairi wa kiarudhi. Kwa hivyo, kuna haja ya kutafiti kuhusu sifa za kimuundo katika ushairi huru hasa diwani huru ya Rangi ya Anga.

\section{Tahakiki za Maandishi}

Tafiti mbalimbali zimefanywa kuhusu ushairi huru wa Kiswahili kwa kuangazia maudhui, fani na dhima. Dhamira ya utafiti huu ni kuangazia sifa za kimuundo katika mashairi huru hasa katika diwani huru ya Rangi ya Anga. Onyancha (2016) alihakiki changamoto za mikondo mipya ya utunzi wa mashairi ya Kiswahili kwa kutumia nadharia ya kimtindo. Utafiti wake uliangazia mikondo mipya ya kutunga mashairi tofauti na maumbo yaliyozoeleka kwa kurejelea mashairi katika Tunu ya Ushairi (2013). Aliangazia mabadiliko ya maneno, kibwagizo na mistari katika ushairi wa kiarudhi pamoja na ushairi huru, picha na arudhi. Alionyesha kuwa mikondo ya kutunga mashairi ya awali inaweza kuendelezwa kwa namna nyingine katika harakati za kujirekebisha na kujiunda upya katika mpito wa wakati. Utafiti huu ni tofauti na utafiti wetu ambao umejikita tu katika mashairi huru katika diwani huru ya Rangi ya Anga; mashairi ambayo yamekiuka sheria za utunzi za wanamapokeo bali si kuziendeleza. Mshairi huru ana uhuru wa kuwasilisha mawazo yake bila kufungwa na sheria za kiarudhi. Hata hivyo, utafiti wake ni wa manufaa kwetu hasa katika kuangazia sifa za kimuundo za mashairi katika diwani teule.

\section{Misingi ya Nadharia}

Nadharia ambazo ziliupa msingi utafiti huu ni nadharia ya uhakiki ya umuundo na nadharia ya uhakiki wa kimtindo. Kutokana na nadharia ya uhakiki ya umuundo kusisitiza mahusiano baina ya vipengele vya kazi ya kisanaa bila kuwa na urejelezi wa nje, nadharia ya uhakiki wa kimtindo imetumika kufidia udhaifu huo. Nadharia hii inarejelea muktadha wa matumizi ya lugha ili kuleta maana halisi na kamili katika fasihi. Mihimili ya nadharia hizi imetumiwa kuhakiki na kutathmini sifa za kimuundo katika diwani teule. Nadharia ya uhakiki ya umuundo inachunguza mahusiano yaliyopo baina ya vipengele vya kimsingi katika lugha, fasihi na nyanja zingine ambapo mifumo mbalimbali ya kimuundo inajengeka. Waasisi wa nadharia hii ni Ferdinand De Saussure na Leonard Bloomfield mnamo mapema karne ya 20. Hali kadhalika, Mulokozi (1989), anasema kuwa mwasisi anayetajwa sana katika nadharia hii ni Saussure (1966), 
aliyependekeza mtazamo wa kiisimu kuhusu lugha kinyume na wataalamu wengine walioshughulikia historia na sura maalum za lugha fulani. De Saussure alivutiwa na miundo inayohimili lugha zote, huku akijaribu kuonyesha kwamba lugha zote za ulimwengu zinaweza kutathminiwa kwa kaida maalum za umuundo. Alizua istilahi, 'Langue' na 'Parole' kuelezea dhana ya lugha. De Saussure aliegemea sana dhana ya 'langue'. Nadharia hii huangalia namna sehemu mbalimbali za kazi za sanaa zilivyofungamana. Hii ina maana kwamba neno halihitaji urejelezi wa nje ili lipate maana na kazi yenyewe ilivyoundwa ndivyo hutoa maana. Jinsi maana imeumbwa ina dhima kubwa kuliko maana yenyewe.

Kutokana na mtazamo wa De Saussure wa kiisimu kuhusu lugha, mhakiki Leech (1969), anahakiki ushairi kwa kuzingatia nadharia ya umuundo. Anasema kazi ya fasihi haiwezi kueleweka vyema bila kuwepo na ufahamu wa lugha ambayo ni kipengee muhimu katika kuwasilisha mawazo na hisia za mtunzi. Leech anasisitiza kwamba lugha ya kishairi hujikita katika vitengo vitatu: fonolojia na grafolojia, umbo na semantiki na viwango hivi huingiliana ili kujenga muundo. Kutokana na mtazamo wake, inabainika kwamba kuna mtagusano baina ya sauti, silabi, viambishi, mofimu, maneno na sentensi. Sauti maalum zinaweza kutumika kuunda maneno ambayo yanaweza kurudiwa kujenga muundo fulani. Leech anaangazia vilevile matumizi ya viakifishi na mpangilio wa sentensi ili kujenga aya. Mtunzi anaweza kuamua kutumia viakifishi ama akose kuvitumia kutegemea maneno aliyoyatumia. Haya yote yanajenga muundo wa shairi. Kwa mujibu wa Leech na Short (1981), ujumi au urembo wa muundo wa matini ndio huvutia msomaji.

Mfuasi wa Ferdinand De Saussure anayetambulikana zaidi ni Jonathan Culler. Culler (1975) alitilia mkazo vipengee vifuatavyo vya umuundo katika ushairi:

i. Ushairi ni ishara zinazojisimamia bila kudhibitiwa na mtunzi.

ii. Ushairi ni maarifa ambayo hayahitaji ufasiri wa kibinafsi kutoka kwa msomaji. iii. Ufasiri wa ujumbe katika ushairi unafaa kuegemea matini wala sio urejelezi wa nje. Ufasiri huu unafaa kuwa halisi.

iv. Msomaji wa ushairi ni mpokeaji tu si mhusika mtendaji katika utunzi wala ufasiri wa maana apendavyo.

v. Ushairi unahusu upangaji wa maneno kwa utaratibu; maneno ambayo huchagizana kimaana. Kwa hivyo mfasiri wa ujumbe anafaa kuangazia mahusiano yaliyopo baina ya maneno/mafungu ya maneno.

vi. Ufasiri wa maana utategemea tajriba ya msomaji katika usomaji wa mashairi. Kwa hivyo, atatoa maana ya kisemantiki.

Naye Ntarangwi (2003), anasisitiza kwamba lugha haiwezi kufanya kazi bila kuathiriwa na mambo mengine kama vile mazingira. Kwa hivyo, ili kufidia upungufu wa umuundo, nadharia ya uhakiki wa kimtindo imetumika pia. Nadharia hii imerejelea muktadha wa matumizi ya lugha ili kuleta maana halisi na kamili (jinsi vipashio hivyo vya lugha vinavyofanikisha uwasilishaji wa ujumbe) kupitia ushairi wa Rangi ya Anga. Namna mtunzi wa ushairi anavyotumia lugha huathiri uelewekaji wake kwa hadhira.

Nadharia ya uhakiki wa kimtindo iliasisiwa na Coombes (1953). Kulingana naye, mhakiki lazima azingatie mambo yanayofanya kazi fulani ivutie, mathalan, taswira, uteuzi wa maneno, urari wa vina na mahadhi ya ushairi. Mwandishi lazima aonyeshe umaarufu wake katika matumizi ya lugha wala si maudhui tu. Kulingana na Crystal na Davy (1969), mtindo unaweza kufafanuliwa kwa njia nne kuu: kwanza, mtindo ni tabia ya mtu kuhusu matumizi yake ya lugha. Pili, mtindo ni jinsi jamii au kikundi fulani cha watu wanavyotumia lugha katika kipindi fulani. Tatu, mtindo ni tabia ya kueleza mambo katika mazingira fulani. Nne, kimsingi mtindo umekuwa ukihusishwa na fasihi kama maandishi "mazuri," yenye sifa bora na yenye umaridadi.

Nadharia ya uhakiki wa kimtindo ina viwango mbalimbali ambavyo humwezesha mchanganuzi wa matini ya kifasihi kuvizingatia ili kutekeleza uhakiki kwa njia ifaayo. Kulingana na Leech 
(1969), kuna aina mbalimbali za ukiushi wa kaida za matumizi ya lugha ambao hutokea katika mtindo wa ushairi. Viwango vya ukiushi ni kama ifuatavyo: ukiushi wa kimsamiati/kileksia, kisarufi, kilahaja, sajili, kifonolojia, kigrafolojia, kisemantiki na kimapisi/kihistoria. Mawazo haya yameungwa mkono na Simpson (2004) na Wamitila (2008).

Kutokana na ufafanuzi wa nadharia ya uhakiki ya umuundo na nadharia ya uhakiki wa kimtindo, vipengele vilivyosaidia katika utafiti wetu ni kama vifuatavyo:

i. Viwango vya kimofolojia na kisintaksia ni msingi mkuu wa lugha unaodhibiti matumizi ya lugha yenyewe. Mhimili huu umetumika katika utafiti wetu kuangazia ukiushi wa urefu wa sentensi. Aidha, namna matumizi ya maneno maalum na teule huleta ujumi wa ushairi.

ii. Matumizi ya kihomolojia yaani maumbo ya picha na ya kipekee huonyesha umilisi wa mtunzi katika kuelezea hisia zake. Katika utafiti wetu, mhimili huu ulichangia pakubwa katika kuchunguza matumizi ya michoro katika diwani teule.

iii. Vipashio mbalimbali vya muundo huingiliana na kujengana. Mhimili huu ulichangia katika kutambua vipashio vilivyojenga mashairi mbalimbali na mahusiano yake. Kwa mfano, mizani ya mapigo huweza kudhibiti wizani ambayo huainishwa kwa kuzingatia urefu wa mistari, uteuzi wa maneno na jinsi maneno yanavyohusiana yenyewe kujenga mistari, aya na shairi zima.

\section{Mbinu za Utafiti}

Muundo wa utafiti huu ni wa kimaelezo. Sifa za kimuundo ambazo zimechunguzwa ni: matumizi ya idhini nyingi za kishairi, matumizi ya lugha ya kimuziki, muundo wa ushairi unaathiri upokezi wa ujumbe, muundo wa ushairi huathiriwa na vipengele mbalimbali, utumiaji michoro kuwasilisha ujumbe maalum na matumizi ya mistari yenye urefu tofauti. Utafiti huu ulifanywa kupitia usomaji wa kina maktabani na mahojiano nyanjani. Sampuli ya kimaksudi ilitumiwa kupata data ya kimsingi katika diwani huru ya Rangi ya Anga. Vilevile, mashairi teule yaliteuliwa kimaksudi ili kurejelea sifa za kimuundo. Aidha, mbinu ya mahojiano ilitumika kukusanya data kutoka kwa mtunzi wa diwani teule. Kithaka wa Mberia, ambaye aliteuliwa kimaksudi kutokana na utunzi wake wa mashairi huru kwa mikondo ambayo haijazoeleka katika utunzi. Kusudi kuu la kumteua ni kupata data ya kuaminika na ya asili kuhusu matumizi yake ya vipengele vya kimuundo katika diwani huru ya Rangi ya Anga. Hojaji ilitumika kama mwongozo wa mahojiano. Data ya upili ilitokana na uteuzi wa kimaksudi wa tasnifu, vitabu, majarida na tahakiki mbalimbali.

\section{Uchanganuzi wa Data}

Muundo wa shairi huangazia vile shairi limepangwa na mahusiano baina ya elementi. Makala hii imechanganua sifa za kimuundo katika diwani huru ya Rangi ya Anga.

Sifa zifuatazo za kimuundo zimechanganuliwa:

i. Matumizi ya idhini nyingi za kishairi.

ii. Matumizi ya lugha ya kimuziki

iii. Muundo wa ushairi unaathiri upokezi wa ujumbe.

iv. Muundo wa ushairi huathiriwa na vipengele mbalimbali.

v. Utumiaji michoro kuwasilisha ujumbe maalum.

vi. Matumizi ya mistari yenye urefu tofauti.

\section{Matumizi ya Idhini nyingi za Kishairi}

Mashairi huru si kazi iliyoandikwa kinathari kama wasemavyo wanamapokeo. Kwa mfano, Abedi (1954) anayesema kwamba mengi kati ya mashairi ya kisasa yanaelekea kupunguza thamani ya mashairi ya Kiswahili mbele ya lugha nyingine. Aidha, wafuasi wengine wa ushairi wa kimapokeo kwa mfano, Abdilatif Abdalla, Shihabuddin Chiraghdin, Hassan Mwalimu Mbega, Ahmad Sheikh Nabhany na wengineo wanaunga maoni haya mkono kwa kuonelea kwamba ushairi umekosa mwelekeo na kwamba kwa kuendeleza maumbo mapya, ladha ya ushairi inapotea na 
hatimaye utanzu wa ushairi utatoweka na kitakachotokea ni utanzu wa nathari unaotumia lugha kwa ufupi.

Washairi wa kisasa wana kibali cha kuwasilisha mawazo yao kwa namna huru bila kufungwa na mishororo na urari wa vina na mizani. Mtunzi yeyote yule hatungi kutokana na ombwe tupu bali kutokana na msukumo au ilhamu fulani; jambo ambalo linaathiri namna anavyowasilisha kazi yake. Kwa hivyo, mtunzi hawasilishi kazi kiholela tu. Ingekuwa hivyo, basi kila mtu angekuwa mtunzi wa ushairi huru. Mshairi ana uhuru wa kupanga mawazo yake jinsi atakavyo kusudi afanikishe uwasilishaji wa maudhui kwa namna atakavyo na kwa njia ya ubunifu. Utafiti huu unadhihirisha ya kwamba, mtunzi ana kibali cha kuzingatia yafuatayo:

i. Matumizi ya mafungu ya maneno

ii. Matumizi ya mishata

iii. Mipangilio mbalimbali ya mistari

iv. Mpangilio wa maneno au sauti kimaksudi

v. Matumizi ya lugha ya mkato na yenye mnato

\section{Matumizi ya Mafungu ya Maneno}

Katika mahojiano yangu na Kithaka wa Mberia kuhusu matumizi yake ya muundo katika diwani huru ya Rangi ya Anga, yaliyoandaliwa ofisini mwake, Chuo Kikuu cha Nairobi, alisisitiza kuwa diwani teule imezingatia muundo wa kimaksudi kutokana na mdundo wa muziki ulio katika fikra za mtunzi. Mashairi yaliyopo katika diwani ya Rangi ya Anga (2014) yamewasilishwa kwa namna ya mafungu. Kwa mfano, katika shairi la 4 (uk. 33). Mshairi anasema:

Kutoka anga la kiangazi

Dhuluma

Zinanguruma kwa nguvu

Na kubabaisha mabonde

Na mawanda na vilima

Mithili mto ulioghafirika

$\mathrm{Na}$ kuasi kingo kwa hasira

Tazama jinsi kondoo

Kuku

Kurumbizi
Pimbi

Mikowe

Mbuzi

Mibono

Na sisimizi

Wanavyotapatapa

Katika dimbwi la joto-

Bwawa la huzuni!

Tazama jinsi sura ya udongo

Ilivyo na alama ya jakamoyo

Kwa kukaangwa kwenye tanuri

Ya jua lenye inda!

Katika shairi hili, mtiririko wa mawazo unadhihirika kutoka fungu moja hadi lingine bila kuzua utata wowote. Idadi ya mistari katika kila fungu inategemea mtiririko wa mawazo katika akili ya mtunzi. Vilevile, idadi hii ya mishororo inategemea namna mtunzi anavyotaka kuwasilisha maudhui yake. Katika kifungu cha kwanza, kuna mistari saba, kifungu cha pili ni kumi na moja huku kifungu cha tatu kikiwa na mistari minne. Katika uwasilishaji wa maudhui, mtunzi anatanguliza wazo la athari ya kiangazi kwa mazingira. Katika kifungu cha pili, anaonyesha viumbe na mimea iliyoathiriwa na kiangazi kama vile kuku, pimbi, mikowe, mbuzi na kondoo. Hatimaye, katika kifungu cha mwisho, anaonyesha namna udongo ulivyoathiriwa na makali ya kiangazi.

Wakati mwingine, mtunzi amewasilisha kazi yake katika fungu moja tu. Kwa mfano, katika shairi la 4 (uk. 6). Anasema:

Wewe mwenye sauti nyororo

Na maungo mviringo na laini,

Wewe usonywaye maishani

$\mathrm{Na}$ wenye taasubi ya jinsia,

Wewe ubezwaye unaposema

Na kutwezwa unapotenda,

Wewe udharauliwaye sokoni

Na kudhalilishwa nyumbani,

Wewe ukemewaye mezani

Na kuonywa vikali chumbani,

Tueleze, huu ni ukweli

Au ni mazigazi machoni

Kwamba mamako mzazi

Anaishi katika tufani

Upepo wa masuto ya daima

$\mathrm{Na}$ fedheha na vitisho vizito

Ukimviringisha kwenye vumbi 
Kwa "kosa lisilostahimilika"

La kuleta duniani watoto wa kike

Badala ya "watoto wastahiki"?

Katika shairi hili, mtunzi ametumia vituo kwa wingi kwa ajili ya kudhibiti usomaji. Matumizi haya yanampa msomaji umakinifu katika usomaji. Mtunzi anawasilisha maudhui ya dhuluma dhidi ya jinsia ya kike. Wanawake wanabezwa katika usemi, wanavuliwa mamlaka, wanadharauliwa sokoni na kudhalilishwa sokoni. Aidha, uwasilishaji huu unaonyesha kiwango cha juu cha ubunifu cha mtunzi na kuondoa uchovu wa kusoma mashairi yanayozingatia muwala wa beti.

Mpito wa wakati huathiri pia uwasilishaji wa ushairi. Mabadiliko yaliyopo katika jamii huathiri sanaa pia. Katika utunzi wa awali wa Msimu wa Tisa (2007), mada kuu katika kila shairi ni dhahiri ilhali katika diwani ya Rangi ya Anga (2014), aliyoiandika baada ya miaka saba, anampa msomaji nafasi ya kuchunguza, kutambua mada na kuchambua maudhui yanayojitokeza katika mashairi yake. Mtunzi ana uhuru wa kupanga kazi yake kiaya kutegemea mada kuu na vimada vyake ambavyo vinadhihirika kupitia maelezo. Ushairi wa diwani ya Rangi ya Anga ni kielelezo bora cha ushairi wa kisasa ambao unadhihirisha ubunifu huu wa mtunzi. Mtunzi amegawa ushairi huu katika mada tatu kuu: Rangi ya Anga, Wanda na Zimwi la Theluji. Kwa mfano, katika shairi la 1 (uk. 2), mshairi ametanguliza ushairi wake kwa kusema:

Baina ya wawili maishani

Uzito wa safari

Ungelingana kama shilingi kwa ya pili,

Utulivu kwenye kumbi za nyoyo

Ungekuwa wa mizani sawa,

Singejipa wadhifa wa mgambo

Singepiga pembe ya swala pala

Kuwakutanisha wanakijiji;

Moyoni mwangu

Hakungekuwa na ilihamu

Ikiniwashawasha kwa utukutu

Kama majani ya mpupu;

Mwashomwasho usiokupwa

Haungekuwa ukirusha mawimbi

Yakibeba mvumo wa sauti

Ukisema kwa uradidi:

Himahima shika pembe

Piga mbiu ya mgambo!
Katika kifungu hiki, mshairi ametanguliza kwa kuteka makini ya msomaji ya kwamba kuna jambo la dharura analolipasha kwa wanajamii. Katika mada ya kwanza, ameangazia dhuluma dhidi ya jinsia ya kike. Kila aina ya dhuluma imeangaziwa kwa kuzingatia mada ndogondogo zilizofafanuliwa katika mafungu. Mtunzi ametumia mbinu ya masimulizi kuwasilisha kazi yake ili msomaji aelewe ujumbe kwa urahisi. Kwa mfano, katika shairi la 2 (uk. 2). Mtunzi anasema:

...Vilele vya milima

Na mahindi ya bahari,

Mwesomweso wa nyota

$\mathrm{Na}$ tumba la mbalamwezi,

Bila kupigana mieleka

Kwenye ukumbi wa ugambi

Zinakubaliana kwa pamoja

Kwamba kuwa navyo mkononi

Ni upini wa upanga mkali

Mkononi mwa mwanamume...

Kutokana na kifungu hiki, mtunzi anaangazia suala mojawapo la dharura ambalo ni taasubi ya kiume inayowapa wanaume nafasi ya kurithi rasilmali katika jamii (ardhi yenye rotuba, mimea iliyonawiri na mazao tele, mifugo na vitu vinginevyo) huku wanawake wakipuuzwa. Inadhihirika wazi kwamba, mafungu haya hayatokei kisadfa bali ni kimaksudi kutokana na mdundo au muziki ulio katika fikra za mtunzi.

\section{Matumizi ya Mishata}

Kulingana na Lutomia (2017: 6), mishata ni mistari ambayo haijitoshelezi kimaana; hutegemeana. Maana yake hukamilika katika mistari inayofuatia. Katika diwani teule, mistari hii imetokea kimaksudi sio kiholela. Inapotumika katika ushairi inaonyesha hali halisi ya mazungumzo japo kwa njia ya mkato na mnato. Mtunzi yu makini kupanga mistari yake kwa namna ambayo itakuwa rahisi kwa msomaji kuufasiri ujumbe unaokusudiwa. Kwa mfano, mishata imetumika kuwasilisha masimulizi yaliyo katika akili ya mtunzi kishairi badala ya kutumia lugha nathari ambayo inatumika katika riwaya au hadithi. Haya yanadhihirika katika diwani ya Rangi ya Anga shairi la 'Rangi ya Anga' (uk. 13). Mshairi anasema:

Walikuwa wachanga

Katika kivuli cha shule 
Hisia za ujana zilipovutana

Mithili ya sumaku na chuma;

Nyoyo zikalainika

Miili ikaitika mwito mkali-

Moto wa maumbile uliwaka

Na kisha kuzimika

Mithili mwako mbugani

Utupao ndimi angani

Na kisha kukata roho

Baada ya wingu la mvua

Kupasuka katika mbingu

Mmoja wa wawili washiriki

Aliendelea na mwendo shuleni

Akakusanya vyeti vya maana

Na kupata jina adimu nchini,

Leo, asubuhi anakula mayai

$\mathrm{Na}$ asali na siagi ya thamani,

Anavalia mavazi ya kuteua

Na kulalia godoro la gharama,

Anatumia fenicha ya mvuli

$\mathrm{Na}$ kulalia matakia ya veliveti...

Katika mifano hii, mtunzi anatumia mishata kutusimulia maisha ya wanagenzi wawili waliozini wangali shuleni. Anaangazia mwanzo wa mahusiano yao na tamati yao. Kijana wa kiume hakuathirika vibaya manake maisha yake yaliendelea na kunawiri ilhali ndoto nzuri za usoni za kijana wa kike zilikatizwa na ujauzito. Mpangilio huu unaondoa uchovu wa kusoma kazi iliyoandikwa kwa lugha nathari.

\section{Mipangilio mbalimbali ya Mistari}

Mtunzi ametumia mipangilio mbalimbali ya mistari katika diwani huru ya Rangi ya Anga. Mshairi anaweza kupanga mistari kwa kurudia vitenzi maalum kwa kusudi la kusisitiza ujumbe fulani. Katika shairi la 10 (uk. 14), mtunzi anasema:

...Banati alipotimuliwa, alitimuka

Alipotimuka, aliteguka

Alipoteguka...

Katika kifungu hiki, mtunzi anasisitiza ujumbe kwa kurudia vitenzi, 'timuka' na 'teguka' katika kauli tofauti ili kuonyesha namna banati alivyotimuliwa kutoka shuleni baada ya kuwa mja-mzito.

Hali kadhalika, mtunzi anaweza kupanga mistari kwa namna ya kuashiria kasi ya kitendo na hisia zinazoambatana na kitendo hicho. Kwa mfano, katika Rangi ya Anga, (uk. 14). Mtunzi anasema:

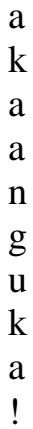

Kutokana na kifungu hiki, mtunzi ameonyesha namna banati alivyokumbana na majanga baada ya kuwa mja-mzito. Sentensi sahili, 'akaanguka' imegawika kisauti na kuandikwa kutoka juu hadi chini badala ya kutoka kushoto hadi kulia. Jambo hili linajenga taswira katika akili ya msomaji kuonyesha kuanguka kwa mwendo wa kasi na kishindo. Hisia za uchungu zinajengeka katika akili ya msomaji kuhusu matokeo ya mahusiano ya kimapenzi baina ya vijana yanayoathiri kwa pakubwa jinsia ya kike. Aidha, kule kugawa sauti kunaleta dhana ya msisitizo wa kitendo hicho.

Mistari vilevile imepangwa kuegemea kulia kwa kusudi la kutoa wazo na jibu. Kwa mfano, katika diwani ya Rangi ya Anga, shairi la 6, (uk. 10). Mtunzi anasema:

...Hiyo

Si taasubi

Ni desturi na mila!

Hizo

Si nyanyaso

Ni kanuni za jadi!

Hizo

Si dhuluma

Ni kaida za utamaduni!

Mpangilio huu unatupa taswira ya ukinzani uliopo baina ya utamaduni na haki za binadamu katika jamii hasa haki za jinsia ya kike. Mtunzi anaonyesha namna mwanamke, ambaye ana shahada ya uzamili anaunga mkono utamaduni unaomkandamiza mwanamke. Anashadidia ya kwamba taasubi ya kiume inapotawala katika jamii ni desturi na mila ambazo zinafaa kufuatwa wala si nyanyaso au dhuluma. Kauli hizi zinaleta hisia za chukizo kwa wanajamii, wakiwemo wanawake 
ambao bado wanaunga mkono tamaduni kandamizi. La kuchukiza zaidi ni kwamba hata wanawake wasomi hawajitokezi bayana kutetea wanawake wanaokandamizwa badala yake wanaunga mkono taasubi ya kiume. Isitoshe, vitenzi, 'si' na 'ni' vimetumika kuleta dhana ya msisitizo. Kwa hivyo, inabidi msomaji atilie mkazo maneno hayo katika usomaji wake. Mtunzi pia amepanga mistari yake kuonyesha ukinzani uliopo katika jamii kuhusu suala la taasubi kwa kubainisha sehemu ya kwanza na ya pili katika mstari mmoja. Mshairi ametumia mbinu ya tabaini katika uundaji wa mistari kuonyesha mtazamo wa wanajamii kuhusu masuala ya kijinsia. Kwa mfano, 'Hiyo si taasubi' (sehemu ya kwanza) 'Ni desturi na mila' (sehemu ya pili). Kwa hivyo, muundo huu unaambatana na mtindo kiumbuji kuwasilisha ujumbe kutokana na matumizi ya 'si' na 'ni' kuonyesha mbinu ya tanakuzi. Mistari hii pia imepangwa kimaksudi kuunda ruwaza fulani. Kusudi kuu ni kuvuta makini ya msomaji.

\section{Mpangilio wa Maneno au Sauti Kimaksudi}

Jambo moja ambalo linadhihirisha ubunifu wa mtunzi katika mashairi teule ni mpangilio wa maneno na sauti. Mtunzi anazingatia mitindo mbalimbali ya kupanga maneno yake kutegemea ujumbe anaouwasilisha na mapigo au mahadhi anayotaka kuzua. Mpangilio huu pia hudhibitiwa na wizani ya ushairi wake. Kwa mfano, mtunzi anaweza kuamua kutumia mtindo wa kurudia neno fulani mwanzoni mwa mistari kadhaa ili kusisitiza ujumbe fulani. Kwa mfano, katika shairi la 'Zimwi la Theluji' (uk. 54). Anasema:
...Lilimfikia malishoni
Na kwa ujuzi na hila
Kumtia kitanzi pembeni
Kitanzi madhubuti
Sio cha kamba ya mgoza
Sio cha katani au mkonge
Sio cha mbuyu au mfungate
Bali cha chuma cha pua...

Kutokana na kifungu hiki, mshairi ametumia fungu la maneno, 'sio cha' kudumisha mahadhi ya ushairi na kusisitiza ujumbe kwa msomaji. Mtunzi anasisitiza jinsi wakoloni walitumia hila kuwatawala Waafrika. Waliwahadaa, sio kwa kuwatia kitanzi cha kamba, katani au mbuyu bali kuhadaa nafsi zao. Katika kifungu hiki pia kuna usambamba wa kategoria za maneno. Kwa mfano, katika kifungu hiki, mstari wa 6 na 7 unazingatia mpangilio wa kitenzi, kihusishi, nomino, kiunganishi na nomino.

Mshairi pia anaweza kuandika maneno katika orodha ili kutenganisha orodha na maelezo. Kwa mfano, katika ushairi wa Rangi ya Anga, (uk.46). Anasema:

...Aulize waulizwa
Huku kuhamahama
Ili kuanzisha upya konde
Kunaleta shibe gani
Kuliko shamba aushi
La kabeji
Sukuma-wiki
Tamata
Mbiringanya
Pilipili-mboga
Mpunga
Mtama
Mahindi...

Kutokana na kifungu hiki, mshairi ameorodhesha mimea (mtama, mahindi na mimea mingineyo) ambayo hupandwa na wakulima wanaoendeleza kilimo cha lima-hama-lima.

\section{Matumizi ya Lugha ya Mkato na yenye Mnato}

Ushairi katika diwani teule umetumia lugha ya mkato na inayovuta hisia za msomaji. Kulingana na Mulokozi (1989: 1), ushairi ni sanaa ya lugha inayoonyesha au kueleza jambo, wazo au tukio kwa namna inayovuta hisia kutokana na mpangilio maalumu wa maneno yaliyoteuliwa, kauli za mkato, picha na tamathali na inayowasilishwa kwa hadhira kwa njia ya nyimbo au maandishi. Katika diwani ya Rangi ya Anga, mtunzi ametumia lugha ya mkato kwa wingi. Kwa mfano, katika shairi la 7 (uk. 10). Mshairi anasema:

Sikiza sauti ya mzazi

Ambayo inakuja juu

Kama wimbi la sumu

Ikimimina kemikali kali

Katika akili asubuhi: Usicheke kiholela

Kama wewe ni mwanamke! Nyamaza! Futa machozi! Ushindwaye na madogo 
Kama mavazi yako

Ni kamisi na rinda!

Katika kifungu hiki, mshairi anawasilisha mawazo yake katika kifungu kimoja tu. Anazungumzia taasubi ya kiume inayotawala katika jamii huku akiibusha nafasi ya mwanamke katika jamii. Mwanamke anadharauliwa kwa kuonekana kushindwa na madogo huku kijana wa kiume akitarajiwa kuwa shujaa katika jamii. Mshairi ametumia lugha ya mkato katika kifungu hiki ili kunasa makini ya msomaji. Pia, kukuza fikra za msomaji anapowazia ujumbe ambao mtunzi anataka kuupitisha kupitia shairi hilo. Aidha, kumpatia msomaji motisha ya kuusoma ushairi kwa sababu ya ufupisho wa ujumbe unaopitishwa kwa vifungu vifupi vifupi.

\section{Matumizi ya Lugha ya Kimuziki}

Katika mashairi teule, mdundo usiobainika umetumiwa kujenga wizani na kuathiri kasi ya msomaji. Mdundo huu unadhibitiwa na jinsi mawazo yanavyotiririka katika akili ya mtunzi. Kwa hivyo, hauzingatii urari wa mizani katika kila mstari wa shairi. Kwa mfano, katika shairi la 'Wanda' (uk. 28). Mshairi anasema:

Joto (silabi 2)

Linapeperusha bendera Linatawala kwa maringo

Na kiburi,

Linanyanyasa tosi

Na macho,

Harara inachokoza kwapa (silabi 9)

(silabi 9)

(silabi 4)

(silabi 7)

(silabi 3)

(silabi 10)
Na kukereketa migongo... (silabi 9)

Katika kifungu hiki, idadi ya silabi inahitilafiana kutoka mstari hadi mwingine. Kwa hivyo, muziki haudhibitiwi tu na urari wa mizani. Mapigo maalum yanabainika kutokana na namna amepanga maneno na sauti kwa namna teule ili kuzua mahadhi maalum; jambo ambalo linaathiri toni na wizani wakati wa usomaji. Katika shairi lili hili, kifungu cha pili, wizani na toni imetumika kuathiri hisia za msomaji pia. Mshairi anasema:

Tazama!

Tazama mayowe kimya

Yakiwayawaya angani

Na kwa ukaidi wa alasiri

Kutisha majani na maua

Na kubabaisha mijusi na nyuki

Mshairi anaangazia makali ya jua kwa mimea, wanyama na wadudu. Anatumia uradidi wa neno, 'tazama' mwanzoni mwa kifungu hiki. Matumizi haya yanazua muziki wa aina fulani katika akili ya mtunzi na ya msomaji pia.

Isitoshe, kutokana na ufahamu ya kwamba Kiswahili ni lugha ya Kibantu, mdundo wa mashairi teule unalingana na mdundo wa nyimbo za Kibantu ambazo zipo chini ya utanzu wa ushairi simulizi, kwa hivyo ushairi huu unaweza kuimbika. Kwa mfano, kuna ulinganifu wa kimuundo katika wimbo wa Pole Musa (Equator Sound Band) kama ulivyonukuliwa na King'ei na Kisovi (2005: 132) na shairi la 'Wanda' katika diwani ya Rangi ya Anga, (uk. 35).

\section{Pole Musa (Equator Sound Band)}

"Musa nimevumilia sana...

...Kupigwapigwa kama mimi punda

Na sura yangu imeharibika...

...Taabu... taabu... taabu...

Kweli Musa uliponioa

Sura yangu

Haikuwa na alama

Hata moja

Nilikuwa na afya nzuri

Nilinona kama ngoima

Ya Kirige

\section{Rangi ya Anga (uk. 35)}

"Kama si makali ya panga

Kwa ujeuri na ukatili

Kuhujumu mashina ya miti,

Kingo za mito na vijito

Miinuko ya milima

Na miteremko ya mabonde

Bado yangekuwa na ng'ao

Za kulinda afya ya udongo

Na ile ya wakazi wake lukuki

Dhidi ya hasira inayotiririka

Ya mgeni mwekundu 


\author{
Oye oye tafadhali Musa \\ Tafuta bibi atakayeweza \\ Kuvumilia hizo tabia zako \\ Ukinywa pombe \\ Wamnywia yeye \\ Ukinywa munyeke \\ Wamnywia yeye \\ Pole... pole... pole..."
}

Isingekuwa kazi ya kwato

Na ushirikiano wa ndimi na taya

Kugofua kinga za udongo

Hii damu ya ardhi adimu

Leo isingekuwa ikitiririka

Wala mwili wake azizi

Kuwa na majeraha mabichi"
Kutokana na mafungu haya, kama vile ridhimu ilivyo muhimu katika nyimbo za Kibantu, vilevile, imechukua nafasi kubwa katika diwani teule. Zaidi ya hayo, hubadilika kutoka ushairi mmoja hadi mwingine kutegemea ujumbe unaowasilishwa na lugha itumikayo. Ridhimu katika shairi hili si taratibu yaani haipangwi kwa njia maalumu kama ilivyo katika ushairi wa kimapokeo. Aidha, haidhihirishwi kwa kuwepo kwa vina, viwe vya kati au vya mwisho badala yake mapigo yake yana mtiririko maalum wa mawazo ya mtunzi yanayojenga ridhimu hiyo. Katika wimbo, 'Pole Musa', silabi za mwisho hazilingani (na, nda, ka, bu...). Haya pia yanadhihirika katika shairi la 'Wanda' (uk. 35) ambapo silabi za mwisho hazilingani (nga, li, ti, to, ma, nde, o...) lakini kuna mapigo maalumu yanayotokea kimuziki na ambayo yanadhihirisha kiwango cha juu cha ubunifu wa mtunzi. Hali kadhalika, nyimbo za Kibantu hugawika katika mafungu. Katika wimbo tulioangazia, mafungu haya yana idadi tofauti ya mistari (4, 7 na 8); jambo ambalo linadhihirika pia katika shairi la 'Wanda' katika Rangi ya Anga (11 na 7). Kwa hivyo, lugha ya kimuziki inaweza kujengeka bila kuzingatia urari wa mistari katika mafungu ya maneno. Isitoshe, lugha hii ya kimuziki huathiri hisia za msomaji kwa urahisi kuliko matumizi ya lugha katika hali ya kawaida.

Suala hili la lugha ya kimuziki katika mashairi linatiliwa mkazo na Kyalo Wamitila katika utangulizi wa Kezilahabi (2008: ii), anaposema kwamba msomaji anapoyasoma mashairi ya Euphrase Kezilahabi anahisi kama anausikiliza wimbo au muziki fulani. Aidha, muziki unaopigwa na ala ya jadi, 'zeze' unamweka msomaji katika muktadha wa Kiafrika anaoweza kujitambulisha nao; muziki anaoweza kuutambua na kuuimba.

\section{Muundo wa Ushairi unaathiri Upokezi wa Ujumbe.}

Katika sifa iliyotangulia ya muundo katika diwani teule, lugha ya kimuziki humwathiri msomaji kwa kutumia wizani na sauti. Athari hizi hutokana na kuwepo kwa mpangilio unaojitokeza kwa kadri mshairi anavyounda kazi yake. Mtunzi wa diwani teule ameuwasilisha ushairi kwa namna mbalimbali; jambo ambalo linaathiri hisia, kasi ya usomaji, toni, sauti na ufasiri wa ujumbe. Mpangilio huu husawiri fikra za mtunzi ambazo zinadhihirika kupitia uandishi. Aidha, mpangilio wa mafungu, mistari, maneno na sauti humwathiri msomaji kwa pakubwa. Kwa mfano, katika shairi la 'Rangi ya Anga' (uk. 8). Mshairi anasema:
Sikiza hiyo sauti
Ulimi ukitoa kauli
Kama upanga mkali
Ukikata mmea mchanga:
Wewe sasa ni mtu mzima
Mshale wa mizani ya miaka
Unaonyesha miaka kumi na minne,
Mbona usiolewe na Mzee Malimali
Kusudi mahari ya ng'ombe na mbuzi
Ituzimie moto wa umaskini
Kama wingu kuu la masika
Likizima ukame nyikani?...

Katika kifungu hiki, mpangilio wa vifungu vyote unaathiri toni na sauti ya msomaji. Mshairi anatanguliza kwa kuonyesha kauli za msimulizi kisha anataja maneno yaliyosemwa na mnenaji ambaye tunakisia ni mzazi wa kiume akimshurutisha bintiye kuolewa na mzee kusudi wapate mahari waepuke umaskini. Mpangilio wa mistari katika sehemu ya kwanza na ya pili unamlazimu msomaji abadili sauti ili kuonyesha kwamba ni kauli za watu wawili tofauti. Msomaji atatumia toni ya makaripio katika sehemu ya pili, hivyo atakuwa ameufasiri ujumbe ipasavyo. 
Katika shairi la 'Wanda' (uk. 33), mshairi anaonyesha hali ya kukata tamaa kutokana na uharibifu wa mazingira uliosababishwa na binadamu mwenyewe. Aanasema:

...Ole wangu!
Moyoni
Napekechwa na uchungu:
Wakulima
Wameua miangati,
Wagemaji
Wamechinja mikoma,
Wasonga-mizinga
Wameangamiza mianjate;
Vizazi vya mijunju
Migunga
Mibarao
Na mitanda-mboo
Vimetumbukizwa kaburini...

Katika kifungu hiki, mshairi anawataja waliohusika katika uharibifu huu kupitia mpangilio maalum wa maneno. Athari ya kimazingira haimwathiri tu mtunzi bali msomaji pia. Kuangamizwa kwa miti asili kama vile mitanda-mboo, mianjate, mikoma na mingineyo ni jambo ambalo linaibusha hisia za uchungu. Aidha, msomaji anapolisoma shairi hili atalisoma kwa mwendo wa utaratibu na msisitizo kutokana na takriri sauti, 'wa' iliyotumika mwanzoni mwa mistari kadhaa. Isitoshe, mpangilio wa maneno unaoambatishwa na matumizi ya viakifishi, unadhibiti kasi ya msomaji.

\section{Muundo wa Ushairi huathiriwa na Vipengele mbalimbali.}

Muundo wa ushairi teule si tengemano ila hubadilika badilika kutegemea dhamira, maudhui, matumizi ya lugha, wakati, wahusika na upekee wa mtunzi katika kuwasilisha kazi yake. Kulingana na Mazrui (1988: viii), ulimwengu hubadilika na hivyo utamaduni wa ushairi lazima ubadilike. Naye Mwai (1988: 5), anadhihirisha hayo anaposema kwamba fasihi hubadilika katika umbo na maudhui kwa hivyo ushairi wa Kiswahili sharti uruhusu mabadiliko yanayotokea katika ushairi huru. Maoni haya yana mashiko kwa sababu tangu kuanzishwa kwa ushairi huru, mabadiliko kadhaa ya kimuundo yamedhihirika. Mabadiliko haya hutokea katika utunzi wa mshairi binafsi au baina ya mshairi mmoja na mwingine. Mtunzi Kithaka wa Mberia ametumia miundo anuwai katika ushairi wake ili kudumisha wizani maalum.

Uruwazaji unajitokeza katika mpangilio wa mistari hasa wakati mtunzi anabainisha usemi wa mhusika mwingine. Muundo huu umeshamiri katika shairi la 'Rangi ya Anga' (uk. 7). Mshairi anasema:

Kwa macho dadisi

Tumeona mikwaruzo na kovu

Katika mikono na shingo

Na mashavu na vipaji

Kama kwamba chui aliyeudhika

Amepigana vita vya kulipiza kisasi

Dhidi ya binadamu kizimbani;

Tulipomulika kwa uchunguzi

Jawabu likatugonga masikioni

Kama kikawe cha manati:

Mume amemwadhibu mkewe-

Wanawake ni kama watoto

Usipowarudi hupoteza njia!

Nyuso zetu zilipoingia kunyanzi

Kwa kutoridhika nyoyo

Sauti "ilifafanua"

Mume ana haki thabiti

Anaruhusiwa kumwadhibu mkewe-

Si alilipa mahari!...

Katika vifungu hivi, ili kuwasilisha maudhui ya dhuluma ya kijinsia katika jamii, mtunzi ametumia usemi halisi wa msemaji hasa wa jinsia ya kiume. Hii ni njia moja ya kuvuta makini ya msomaji na kusaidia katika uelewekaji wa ujumbe. Mtunzi ameonyesha namna mwanaume ana kibali cha kumpiga mkewe kwa sababu amelipa mahari na asipomwadhibu atapoteza njia.

Hali kadhalika, mshairi ametumia muundo wa masimulizi katika mashairi mbalimbali ili kuwasilisha ujumbe wake kwa namna ya kuvutia. Masimulizi haya pia yanaangaziwa na Kyalo Wamitila katika utangulizi wa ushairi wa Dhifa (2008: ii), anaposema kwamba ushairi wake umehusisha mbinu za kifasihi simulizi kama utambaji na uganaji katika mashairi mengi na kuonyesha kuwa mashairi ya kisimulizi hayapatikani tu katika tendi na tenzi bali pia katika mashairi mafupi. Aidha, usimulizi pia unadhihirishwa katika shairi la 2 (uk. 49). Mshairi anasema:

Kwa kunyemelea 
Lilikuja zimwi jeupe

Kwanza kwenye upwa

Ambapo upepo

Hupuliza manukato ya chumvi

Kwenye pua za wakazi,

Nyanda ambako

Mipeketo na mikungu

Na mianzi na mikoko

Na mikanju na mivinje

Huishi kwa amani

Pamoja na korongo

$\mathrm{Na}$ kunguru na membe...

Katika kifungu hiki, kiambishi cha wakati uliopita (li) kimetumika katika masimulizi kuelezea mjo wa Waarabu, wamisheni na wakoloni katika nchi ya Kenya. Mshairi ametumia mbinu rejeshi kwa sababu anazungumzia matukio ambayo yalitendeka awali, hivyo kumkumbusha msomaji kuhusu historia ya Kenya. Lakini wakati mshairi anatusimulia kuhusu athari za kimazingira katika shairi la 'Wanda' (uk. 28) anatumia wakati uliopo. Anasema:
Joto
Linapeperusha bendera
Linatawala kwa maringo
Na kiburi,
Linanyanyasa tosi
Na macho,
Harara inachokoza kwapa
Na kukereketa migongo...

Katika kifungu hiki, mshairi anatumia hasa wakati uliopo (na). Anataka kudhihirisha ya kwamba athari hizi bado zipo katika jamii. Hii ni njia mojawapo ya kumrejesha msomaji katika matukio halisi.

Isitoshe, mtunzi anaunda sentensi zake kwa namna teule huku akilenga hadhira teule/lengwa. Kwa mfano, katika shairi la 'Wanda' (uk.28). Mshairi anasema:

Jua!

Ewe jua!

Jua la adhuhuri,

Mbona kwa ghadhabu

Unaadhibu chembe za udongo

Kwa halijoto kama ya tanuri?

Jua!
Ewe jua!

Jua la wanda za mikwaju,

Mimea na samaki

Ndege na wadudu

$\mathrm{Na}$ wanyama na mito

Walikutenda ovu gani

Kukuchochea ulipishe kisasi

Kwa ghadhabu pujufu?...

Katika vifungu hivi, mshairi anazungumzia nafsi ya pili (ewe) ili kunasa fikra na hisia za msomaji. Anazungumza na anachokiashiria (jua) kama kipo katika mazingira yake.

Kwa mujibu wa Wordsworth (1797: 52), shairi linafaa kuwasilisha hisia na matukio halisi ya binadamu. Mtunzi ana uhuru wa kujinasibisha na ulimwengu halisi katika kuwasilisha mawazo yake; jambo ambalo linaathiri miundo ya kisintaksia ya ushairi. Kwa mfano, katika shairi la 'Wanda' (uk. 41). Mshairi anasema:

Kibuhuti kinavuma mwangu moyoni

Nakuhurumia, ewe Kereka

Mhisani uliyenilea,

Nawasikitikia, enyi Gitugu

Kamwathu

Kaguma

Kamanyaki

Gaciongo

Gakauni

Kamaindi

Mutino

Nkarini

Ntoroni

Kamajagi

Ntumira

$\mathrm{Na}$ ahali yenu nzima

Kwa jinsi sura zenu

Zilivyojaa vidonda

Na damu mbichi...

Katika vifungu hivi, mshairi anajiweka katika mazingira alimokulia. Ameorodhesha vijiji vilivyomzunguka kama vile Kamaindi na Gakauni na namna vilivyoathiriwa na kiangazi.

Miundo ya kisintaksia pia huathiriwa na madhumuni ya mshairi katika kuwasilisha kazi yake. Kwa mfano, katika shairi la 6 (uk. 8), mtunzi anasema: 
Sikiza hiyo sauti

Ulimi ukitoa kauli

Kama upanga mkali

Ukikata mmea mchanga:

Wewe sasa ni mtu mzima

Mshale wa mizani ya miaka

Unaonyesha miaka kumi na minne,

Mbona usiolewe na Mzee Malimali

Kusudi mahari ya ng'ombe na mbuzi

Ituzimie moto wa umaskini

Kama wingu kuu la masika

Likizima ukame nyikani?..

Katika kifungu hiki, madhumuni ya mshairi ni kuonyesha mtazamo wa jinsia ya kiume kuhusu jinsia ya kike katika jamii. Kwa hivyo, anaegemeza nukuu upande wa kulia ili kudhihirisha wazi makaripio ya baba mzazi kwa mtoto wake wa kike.

\section{Utumiaji michoro kuwasilisha Ujumbe maalum}

Kulingana na Crystal na Davy (1969), matumizi ya sauti na michoro yenye maumbo maalum hudhihirisha ujumi, uhalisia na ufafanuzi zaidi kuhusu ujumbe unaopitishwa na mwandishi katika kazi ya fasihi. Aidha, Pierce (1977: 228) alithibitisha matumizi ya ishara alipoangazia nadharia ya ishara katika kazi ya fasihi. Alisema kwamba ishara huonyesha uhusiano uliopo kati ya kiashiria na kiashiriwa. Alibainisha vitengo vitatu vya ishara, mathalan, ishara halisi ambazo zinaweza kuwa ni sifa, kanuni, desturi au tabia za kitu chenyewe. Pia, ishara ambazo zinahusishwa na viashiriwa. Aidha, ni uhusiano uliopo kati ya kiashiria na kiashiriwa. Katika kitengo cha pili, alibainisha ishara katika vikundi vitatu: picha/mchoro, kielezo cha sifa au tabia na mfumo wa alama zinazowakilisha kundi fulani la mawazo. Maoni haya yanaungwa mkono na Hoopes (1991) anayesema kwamba ishara za picha, kielezo cha sifa na mfumo wa alama zinazowakilisha kundi fulani la mawazo huonyesha namna ishara zinazoonekana zinavyosheheni ujumbe wa kidhahania. Aidha, ishara hizi humsaidia mtafiti kutafakari zaidi kuhusu ujumbe unaowakilishwa na ishara hizo.

Katika sehemu hii tumeangazia ishara za picha katika diwani teule. Michoro ya kimaksudi imetumiwa kuonyesha kasi, hisia na msisitizo wa ujumbe. Katika shairi la 'Rangi ya Anga' (uk. 14), mshairi anasema:

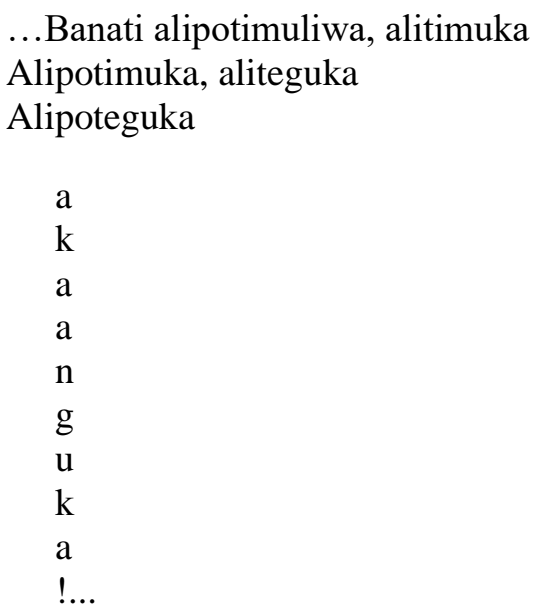

Katika kifungu hiki, mshairi ametuchorea taswira ya mkasa uliomfika kijana msichana aliyepachikwa mimba na mwenzake wa kiume wangali shuleni. Baada ya kufukuzwa shuleni, maisha yake yalikuwa ya dhiki; asiye na mbele wala nyuma; akatumbukia katika shimo la maangamizi. Mshairi anapanga sauti katika neno, 'akaanguka' kutoka juu hadi chini ili kuashiria maangamizi. Isitoshe, anaonyesha kasi ya tukio hilo ambalo huathiriwa na mvuto wa ardhi. Msisitizo wa kitendo cha kuanguka unajitokeza katika kule kutenganisha sauti katika neno hilo. Mchoro huu unaibua hisia za uchungu na huzuni wakati msomaji anapotafakari yaliyomfika banati huyu. Vilevile, unaonyesha hisia za mshairi kuhusu dhuluma anayopitia mtoto wa kike katika jamii.

Mchoro huu umetumiwa kuteka nadhari ya msomaji na kumpa nafasi ya kutafakari zaidi kuhusu ujumbe unaowasilishwa na mshairi. Ni njia moja ya kujiepusha na miundo iliyozoelewa ya beti, hivyo kuondoa uchovu wa usomaji. Isitoshe, mchoro huu hujenga ruwaza ya ushairi kwa kuupa ushairi picha ya pekee na kuonyesha kiwango cha juu cha ubunifu wa mtunzi. Naunga mkono maoni ya Indede (2008: 12) anaposema kwamba ilhamu ya Kithaka wa Mberia ya kuwasilisha mawazo ya ndani kwa matumizi ya picha halisi ili kudhihirisha uwazi wa ayatamkayo, si tu maono mapya katika uvumbuzi wa usanii kisayansi bali pia uvumbuzi wa falsafa mpya ya kisanaa. 


\section{Matumizi ya Mistari yenye urefu tofauti}

Katika diwani huru ya Rangi ya Anga, mtunzi ana uhuru wa kuzingatia urefu autakao kwa kuanzia kwa idadi ndogo ya silabi kuelekea idadi kubwa. Kwa mfano, katika shairi la 'Rangi ya Anga' (uk. 10). Mshairi anasema:

...Hiyo

Hizo

Hizo

$$
\text { Si taasubi }
$$

$$
\text { Ni desturi na mila! }
$$

(silabi 2)

(silabi 5)

(silabi 7)

(silabi 2)

(silabi 4)

(silabi 7)

(silabi 2)

(silabi 4)

Si dhuluma

Ni kaida za utamaduni! (silabi 10)

Katika shairi hili, mpangilio wa mishororo mifupi na mirefu unapunguza kasi ya usomaji na inabidi msomaji asome kwa utaratibu huku akitua baada ya kila fungu la maneno. Urefu wa mistari umedhibitiwa na fikra za mtunzi katika kuwasilisha ujumbe wake kwa njia ambayo itaeleweka na msomaji. Ametumia mbinu za tanakuzi kama vile, 'hiyo si taasubi, ni desturi na mila' kuonyesha kauli kinzani. Vilevile, ametumia tabaini kwa kutumia kisisitizi 'si'. Kwa mfano, 'Hizo si nyanyaso ni kanuni za jadi'. Mbinu hizi zinaonyesha mgogoro uliopo katika jamii kuhusu taasubi ya kiume. Isitoshe, kupitia mpangilio huu na idadi ya maneno katika kila mstari, ujumbe unaopitishwa unasisitizwa. Matumizi haya ya mistari mifupi na mirefu huondoa uchovu wa kusoma na kumpa msomaji hamu ya kusoma shairi zaidi.

Mtunzi pia amegawa mistari yake katika vipande kutegemea dhamira yake. Alama ya mkato (,) imetumika katika kutenganisha vipande. Kwa mfano, katika shairi la 10 (uk. 14). Mshairi anasema:

Banati alipotimuliwa, alitimuka Alipotimuka, aliteguka...

Katika kifungu hiki, mshairi amegawa mistari hii kimaksudi tu katika mistari teule wala si katika shairi zima. Ametumia mtindo huu kuonyesha matokeo ya vitendo. Kutua huku ni muhimu kwa msomaji katika kudhibiti usomaji wake. Isitoshe, kunaonyesha mtiririko wa mawazo ya mtunzi.
Mistari mingine imeundwa kwa kuzingatia neno moja hasa nomino au kiwakilishi. Kwa mfano, katika shairi la 'Wanda' (uk. 33-34). Mshairi anasema:

Kutoka anga la kiangazi
Dhuluma
Zinanguruma kwa nguvu
Na kubabaisha mabonde
Na mawanda na vilima
Mithili mto ulioghafirika
Na kuasi kingo kwa hasira
Tazama jinsi kondoo
Kuku
Kurumbizi
Pimbi
Mikowe
Mbuzi
Mibono
Na sisimizi
Wanavyotapatapa
Katika dimbwi la joto-
Bwawa la huzuni!

Katika kifungu hiki, mshairi ametumia mstari unaoundwa kwa neno moja kuashiria orodha ya vitu. Kusudi kuu ni kutenganisha orodha na maelezo mengine. Ametoa orodha ya viumbe walioathiriwa na joto, mathalan, kuku, kurumbizi, pimbi na viumbe vinginevyo.

\section{HITIMISHO}

Makala hii imehakiki sifa za kimuundo katika diwani teule ya Rangi ya Anga .Imebainika kwamba mashairi teule yana idhini nyingi za utunzi. Aidha, ni mashairi ambayo yanaweza kuimbika kutokana na sifa zake za kimuundo. Mtiririko wa mawazo katika akili ya mtunzi huathiri pakubwa uwasilishaji wake katika utunzi. Wizani inayojitokeza katika ushairi teule hutokana na mtiririko wa mawazo katika akili ya mtunzi. Hali kadhalika, wizani hii huathiri muundo wa kazi yake. Wizani hii hudhibitiwa na mpangilio wa mafungu, mistari, maneno na sauti. Aidha, muundo huu huathiri hisia, toni, kasi na sauti ya msomaji. Katika makala hii pia, mtunzi hajazingatia muundo mmoja katika utunzi wake bali amebadili miundo kutegemea vipengele mbalimbali kama vile: dhamira, maudhui, matumizi ya lugha, wahusika na upekee wa mwandishi. Isitoshe, mtunzi hajafungwa 
na muundo maalum wa muwala wa beti na urari wa mizani katika kila mstari bali ana uhuru wa kuwasilisha ujumbe wake kishairi kwa kutumia michoro na mistari ambayo haina urefu maalum. Kwa hivyo, ushairi teule unaonyesha ubunifu wa kiwango cha juu cha mtunzi wala si maandishi ya kiholela holela yasiyokuwa na mantiki. Aidha, sifa za kimuundo katika ushairi teule ni muhimu katika kuufasiri ujumbe wa mshairi.

\section{MAREJELEO}

Abedi, A. (1954). Sheria za Kutunga Mashairi na Diwani ya Amri. Nairobi: Kenya Literature Bureau.

Coombes, H. (1953). Literature and Criticism. Newyork: Chatto and Windows.

Crystal, D. \& Davy, D. (1969). Investigating English Style. London: Longman Group Limited.

Culler, J. (1975). Structuralist Poetics. Structuralism, Linguistics \& the Study of Literature. London: Routledge.

Hoopes, J. (1991). Peine on Signs: Writings on Semiotic by Charles Sanders Peine. Chapel Hill London: The University of North Carolina Press.

Indede, F. (2008). Mabadiliko katika Umbo la Ushairi na Athari zake katika Ushairi wa Kiswahili: SWAHILI FORUM 15:73-94.

Kezilahabi, E. (2008). Dhifa. Nairobi: Vide-Muwa Publishers Limited.

King'ei, K. \& Kisovi, C. (2005). Msingi wa Fasihi Simulizi. Nairobi: Kenya Literature Bureau.

Leech, G. \& Short, M. (1981). Style in Fiction: A Linguistic Introduction to English Fictional Prose 2nd Edition. Harlow: Longman Group Ltd. ; Essays and Reviews on $19^{\text {th }} \& 20^{\text {th }}$ Century Literature. London: Routledge \& Kegan Paul.

Leech, G. (1969). A Linguistic Guide to English Poetry. London \& New York: Longman.

Lutomia, D. (2017). Mwanga wa Ushairi kwa Shule za Upili. Kakamega: Double Shasa Limited.
Mazrui, A. (1988). Chembe Cha Moyo. Nairobi: Heinemann Kenya Ltd.

Mberia, K. (2007). Msimu wa Tisa. Nairobi: Marimba Publications Ltd.

Mberia, K. (2014). Rangi ya Anga. Nairobi: Marimba Publications Ltd.

Mulokozi, M. (1989). Mgawanyo wa Tanzu na Vipera vya Fasihi Simulizi. Dar es Salaam: TUKI

Mwai, W. (1988). New Trends in Ushairi, Tasnifu ya M.A., Chuo Kikuu cha Nairobi.

Ntarangwi, M. (2003). Gender, Performance \& Identity. NewYork: Africa World Press.

Onyancha, W. (2016). Changamoto za Mikondo Mipya ya Utunzi wa Mashairi ya Kiswahili, Tasnifu ya Uzamili, Chuo Kikuu cha Kenyatta.

Pierce, C. \& Welby, V. (1977). Semiotic and Significs. Canada: Indiana University Press.

Saussure, F. (1966). Course in General Linguistics. Newyork: Mc Graw-Hill.

Simpson, P. (2004). Stylistics. London \& NewYork: Routledge.

Wamitila, K. W. (2008). Kanzi ya Fasihi. Misingi ya Uchanganuzi wa Fasihi. Nairobi: VideMuwa Publishers Limited.

Wordsworth, W. (1797). Lyrical Ballads. London: Methuen \& Company Ltd. 